\title{
Regional Differences in Human Intestinal Drug Metabolism ${ }^{\S}$
}

\author{
(1) Raditya Iswandana, Marina I. Irianti, Dorenda Oosterhuis, Hendrik S. Hofker, \\ Marjolijn T. Merema, Marina H. de Jager, Henricus A. M. Mutsaers, and Peter Olinga
}

Division of Pharmaceutical Technology and Biopharmacy (R.I., M.I.I., D.O., H.A.M.M., P.O.) and Division of Pharmacokinetics, Toxicology and Targeting (M.T.M., M.H.d.J.), Department of Pharmacy, University of Groningen, Groningen, The Netherlands; Faculty of Pharmacy, Universitas Indonesia, Depok, Indonesia (R.I.); Department of Surgery, University Medical Center Groningen, Groningen, The Netherlands (H.S.H.); and Department of Clinical Medicine, Aarhus University, Aarhus, Denmark (H.A.M.M.)

Received July 4, 2018; accepted September 27, 2018

\section{ABSTRACT}

The intestines are key for the absorption of nutrients and water as well as drug metabolism, and it is well known that there are clear differences in the expression profile of drug metabolism enzymes along the intestinal tract. Yet only a few studies have thoroughly investigated regional differences in human intestinal drug metabolism. In this study, we evaluated phase I and phase II metabolism in matched human ileum and colon precision-cut intestinal slices (PCIS). To this end, human PCIS were incubated for 3 hours with testosterone and 7-hydroxycoumarin $(7-\mathrm{HC})$ to examine phase I and phase II metabolism, respectively. Metabolite formation was assessed by high-performance liquid chromatography analysis.
Our results demonstrated that androstenedione, $6 \beta$-hydroxytestosterone, $2 \beta$-hydroxytestosterone, and $7-\mathrm{HC}$ sulfate were predominantly formed in the ileum, while $15 \alpha$-hydroxytestosterone and 7-HC glucuronide were mainly produced in the colon. Moreover, we also observed sex differences in phase II metabolite formation, which appeared to be higher in men compared with women. Taken together, we demonstrated that phase I metabolism predominantly occurs in ileum PCIS, while phase II metabolism mostly takes place in colon PCIS. Moreover, we revealed that human PCIS can be used to study both regional and sex differences in intestinal metabolism.

\section{Introduction}

In addition to the absorption of nutrients and water, the intestines also fulfill an important role in drug metabolism (Doherty and Charman, 2002; Ding and Kaminsky, 2003, Wang et al., 2003). This is illustrated by the high expression level of drug metabolizing enzymes along the intestinal tract (von Richter et al., 2001; Glaeser et al., 2004).

In the human body, xenobiotics are metabolized via various pathways, including phase I and phase II metabolism. Phase I reactions are oxidation, reduction, and hydrolysis processes. These chemical reactions change the biologic activity of the parent compound, rendering it less or completely inactive (Chhabra, 1979). In the intestines, the most abundant group of enzymes belongs to the cytochrome P450 subfamily $3 \mathrm{~A}$. Nonetheless, the expression profile of CYP enzymes differs along the intestinal tract and species differences are apparent (Martignoni et al., 2006). For instance, CYP3A13 is more common in the mouse intestine while CYP3A4/5 is predominantly found in the human small intestine (Martignoni et al., 2006). In addition, it has been demonstrated that the mRNA levels of CYP isoforms diminish along the intestinal tract, with the highest CYP expression in the duodenum and the lowest in the colon (de Waziers et al., 1990; Martignoni et al., 2006). To study metabolic activity, it is key to use suitable substrates. In the last decades, testosterone (TT; Kim et al., 2016; Fig. 1) and 7-hydroxycoumarin

This work was supported by De Nederlandse organisatie voor gezondheidsonderzoek en zorginnovatie (ZonMW)-The Netherlands [Grant 114025003].

https://doi.org/10.1124/dmd.118.083428.

SThis article has supplemental material available at dmd.aspetjournals.org.
(7-HC; Kim et al., 2016; Fig. 1) have been widely used to examine phase I and phase II metabolism, respectively (De Kanter et al., 2002; Choi et al., 2005). TT can be used to study phase I metabolism since this hormone is hydroxylated by cytochrome P450 enzymes (CYP3A, CYP2A, and CYP2B; Arlotto et al., 1991; Honkakoski and Negishi, 1997; van de Kerkhof et al., 2005; van de Kerkhof et al., 2007).

Phase II reactions are conjugation reactions, e.g., glucuronidation and sulfation, that improve the solubility of endo- and xenobiotics (Chhabra, 1979). 7-HC can be used to examine both reactions (van de Kerkhof et al., 2007): glucuronidation of 7-HC is mediated by uridine 5 '-diphospho- $N$-acetylgalactosamine glycosyltransferase (UGTs), while sulfotransferase (SULTs) catalyze sulfation reactions (Wang et al., 2003, 2006).

Until now, most in vitro studies on intestinal metabolism were conducted by using subcellular fractions like microsomes or enterocytes. By using advanced analytical methods, it has been demonstrated that the majority of drug metabolism enzymes can be detected in intestinal microsomes. Moreover, it has been shown that microsomes can be used to investigate enzymatic activity and the intestinal contribution to first-pass drug metabolism (Gröer et al., 2014; Nakamura et al., 2016; Busch et al., 2018). However, microsomes lack membrane transporters as well as low abundant enzyme systems, and the enzymatic activity in microsomes is highly dependent on the isolation method (Emoto et al., 2000a; van de Kerkhof et al., 2005; Peters et al., 2016; Drozdzik et al., 2018).

Recently, it was demonstrated that precision-cut tissue slices can be used to study intestinal drug metabolism (van de Kerkhof et al., 2008).

ABBREVIATIONS: $15 \alpha$-TOH, $15 \alpha$-hydroxytestostrone; $2 \beta$-TOH, $2 \beta$-hydroxytestosterone; $6 \beta$-TOH, $6 \beta$-hydroxytestosterone; 7 -HC, 7 -hydroxycoumarin; CYP, cytochrome P450; hPCIS, human precision-cut intestinal slices; HPLC, high-performance liquid chromatography; PCIS, precisioncut intestinal slices; SULTs, sulfotransferase; TT, testosterone; UGTs, uridine 5'-diphospho- $N$-acetylgalactosamine glycosyltransferase. 


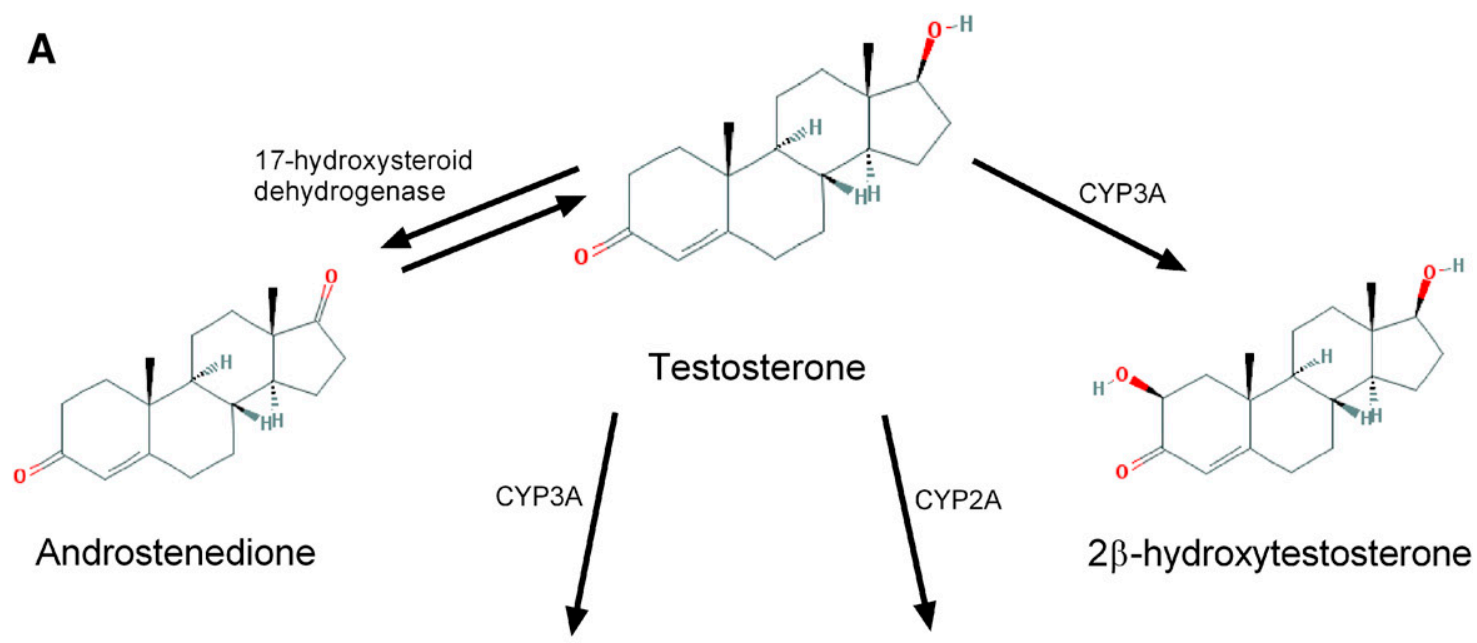

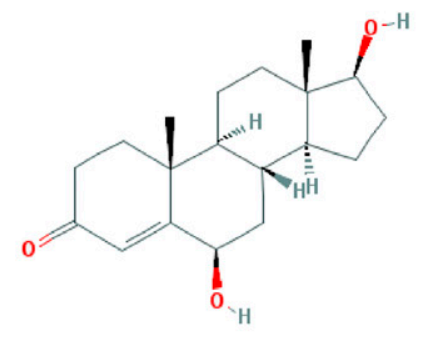

$6 \beta$-hydroxytestosterone

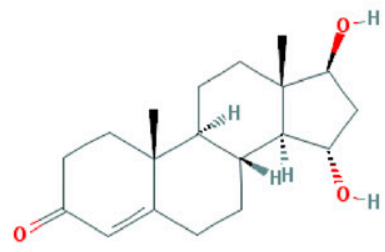

$15 \alpha$-hydroxytestostrone

B<smiles>O=c1ccc2ccc(O)cc2o1</smiles>

7-hydroxycoumarin<smiles>O=C(O)[C@H]1O[C@@H](Oc2ccc3ccc(=O)oc3c2)[C@H](O)[C@@H](O)[C@H]1O</smiles>

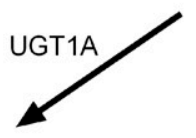
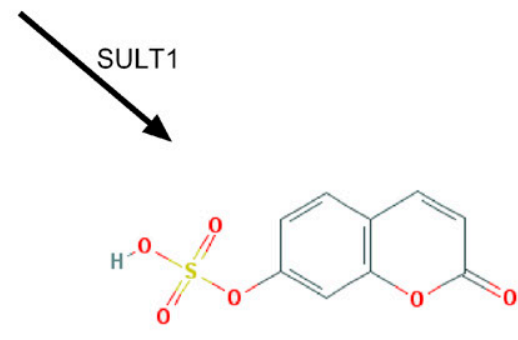

7-HC sulfate

\section{7-HC glucuronide}

Fig. 1. Testosterone and 7-hydroxycoumarin metabolism pathways: (A) Phase I; (B) Phase II.

In this ex vivo model, all the different cells that constitute an organ are maintained in their original environment, allowing for cell-cell and cellmatrix interactions (Poosti et al., 2015). In precision-cut intestinal slices (PCIS) the organization of the intestinal villi and microvilli is well conserved (Pham et al., 2015). Also, the method is highly efficient, since a large number of slices can be prepared from a small tissue sample, and the slices are relatively easy to process (van de Kerkhof et al., 2005). Here, we studied the regional differences in intestinal metabolism by using matched human ileum and colon PCIS focusing on both phase I and phase II metabolism. 
TABLE 1

Characteristics of human PCIS from the human donors

\begin{tabular}{lcc}
\hline Human ID & Sex & Age \\
\hline Patient 1 & M & 64 \\
Patient 2 & M & 52 \\
Patient 3 & M & 88 \\
Patient 4 & F & 79 \\
Patient 5 & F & 65 \\
Patient 6 & M & 64 \\
Patient 7 & F & 81 \\
\hline
\end{tabular}

M, male; F, female.

\section{Materials and Methods}

\section{Chemical}

All chemicals were obtained from Sigma (Zwijndrecht, The Netherlands) unless stated otherwise. Stock solutions of testosterone (TT) and 7-hydroxycoumarin (7-HC) were prepared in methanol and stored at $-20^{\circ} \mathrm{C}$.

\section{Preparation of Intestinal Slices}

Healthy human ileum and colon tissue were obtained following hemicolectomy for adenocarcinoma (see Table 1 for patient characteristics). Use of human tissue was approved by the Medical Ethical Committee of the University Medical Centre Groningen (UMCG), according to Dutch legislation and the Code of Conduct for dealing responsibly with human tissue in the context of health research (www.federa.org), refraining the need of written consent for "further use" of coded-anonymous human tissue. The procedures were carried out in accordance with the experimental protocols approved by the Medical Ethical Committee of the UMCG.

\section{Slicing of Precision-Cut Intestinal Slices}

Preparation of intestinal slices (PCIS) was carried out according to the protocol of de Graaf et al. (2010). In short, intestinal tissue was cleansed by flushing KrebsHenseleit buffer through the lumen and subsequently divided into $2-\mathrm{cm}$ segments. Afterward, intestinal cores were prepared using 3\% (w/v) agarose (Sigma-Aldrich, Steinheim, Germany) in $0.9 \% \mathrm{NaCl}$ at $37^{\circ} \mathrm{C}$ and embedded in an agarose coreembedding unit. Next, PCIS were prepared using a Krumdieck tissue slicer. PCIS had a wet weight of approximately $3 \mathrm{mg}$ and an estimated thickness of 300-400 $\mu \mathrm{m}$. After being sliced, PCIS were directly transferred to Krebs-Henseleit buffer to prevent loss of viability.

\section{Incubation of Slices}

After slicing, PCIS were cultured in 12-well plates containing Williams' Medium E + Glutamax (Gibco, New York, NY) supplemented with $14 \mathrm{mM}$ Glucose (Merck, Darmstadt, Germany), $50 \mu \mathrm{g} / \mathrm{ml}$ gentamycin (Gibco) and $2.5 \mu \mathrm{g} / \mathrm{ml}$ fungizone (amphotericin B; Invitrogen, Paisly, Scotland). Slices were cultured at $37^{\circ} \mathrm{C}$ in an $80 \% \mathrm{O}_{2} / 5 \% \mathrm{CO}_{2}$ atmosphere, while horizontally shaken at $90 \mathrm{rpm}$ (amplitude $2 \mathrm{~cm}$ ). For metabolism experiments, PCIS were incubated with TT $(250 \mu \mathrm{M})$ or $7-\mathrm{HC}(500 \mu \mathrm{M})$ for 3 hours.

\section{Viability}

Viability of PCIS was assessed by measuring the adenosine triphosphate content of the slices using an ATP bioluminescence kit (Roche Diagnostics, Mannheim, Germany), as previously described (de Graaf et al., 2010). Determined ATP values (picomoles) were normalized to the total amount of protein (micrograms) estimated by the Lowry method (BIO-rad RC DC Protein Assay; Bio Rad, Veenendaal, The Netherlands). Results are shown as relative values compared with the related controls.

\section{Metabolite Analysis}

Testosterone. After incubation with testosterone (TT), PCIS and medium were collected and stored at $-20^{\circ} \mathrm{C}$ until further use. Sample extraction and highperformance liquid chromatography (HPLC) analysis were performed as described earlier (van de Kerkhof et al., 2006).
7-Hydroxycoumarin. 7-Hydroxycoumarin (7-HC) and their metabolites do not accumulate in PCIS; therefore the analysis was performed using medium samples only (De Kanter et al., 2002; de Kanter et al., 2005). After incubation, samples were collected and stored at $-20^{\circ} \mathrm{C}$ until use. Levels of $7-\mathrm{HC}$ glucuronide and 7-HC sulfate were determined via HPLC analysis as described previously (van de Kerkhof et al., 2005).

\section{Statistics}

Statistics were performed using GraphPad Prism 6.0 via two-tailed Student's $t$ test and Pearson rank correlation as appropriate. A minimum of three different intestines was used for each experiment, using three to six slices from each intestine per condition. The results are expressed as mean \pm S.E.M. Statistical differences in ATP levels were determined using the values relative to the control values. Differences between groups were considered to be statistically significant when $P<0.05$.

\section{Results}

Viability of Ileum and Colon Slices. Our laboratory previously demonstrated that rat PCIS could be used to study intestinal metabolism in vitro. To gain more insight into human metabolism, we prepared matched ileum and colon PCIS, allowing us to study regional differences in intestinal metabolism. First, we characterized viability of the slices by determining the ATP content. As shown in Fig. 2, the ATP content of human ileum PCIS significantly increased after 3 hour of culture compared with the 0 hour control, after which the levels remained stable. Similar results were obtained with colon PCIS, indicating that intestinal slices remain viable in culture for at least 24 hours.

Assessment of Phase I Metabolism in Ileum and Colon. To study phase I metabolism, we incubated ileum and colon PCIS with TT. As shown in Fig. 3, several testosterone metabolites were formed in both ileum and colon slices, e.g., androstenedione, $6 \beta$-hydroxytestosterone $(6 \beta$-TOH), $15 \alpha$-hydroxytestosterone $(15 \alpha-\mathrm{TOH})$, and $2 \beta$-hydroxytestosterone $(2 \beta-\mathrm{TOH})$. However, there was a clear distinction between the metabolite levels in ileum and colon (Fig. $3 ; n=5$ ). Androstenedione, formed by $17 \beta$-hydroxysteroid dehydrogenase, is the main TT metabolite that can be found in both the ileum and colon with a mean concentration of $9.55 \pm 2.27$ (range 3.0-15.6) and $4.34 \pm 0.96$ (range $2.0-9.3) \mathrm{pmol} / \mu \mathrm{g}$ protein, respectively. $6 \beta-\mathrm{TOH}$ was also found in all patient samples, however, the levels were approximately 4.2-fold higher in the ileum than in the colon (Fig. $3 ; n=5$ ). In addition, $2 \beta$-TOH levels also appeared to be higher in the ileum than in the colon, with a mean concentration of $0.24 \pm 0.11$ and $0.06 \pm 0.01 \mathrm{pmol} / \mu \mathrm{g}$ protein, respectively, although differences were not statistically significant. Regarding $15 \alpha-\mathrm{TOH}$, no differences were observed. Overall, these results suggest that the ileum plays a bigger role in phase I metabolism as compared with the colon (Supplemental Table S1).

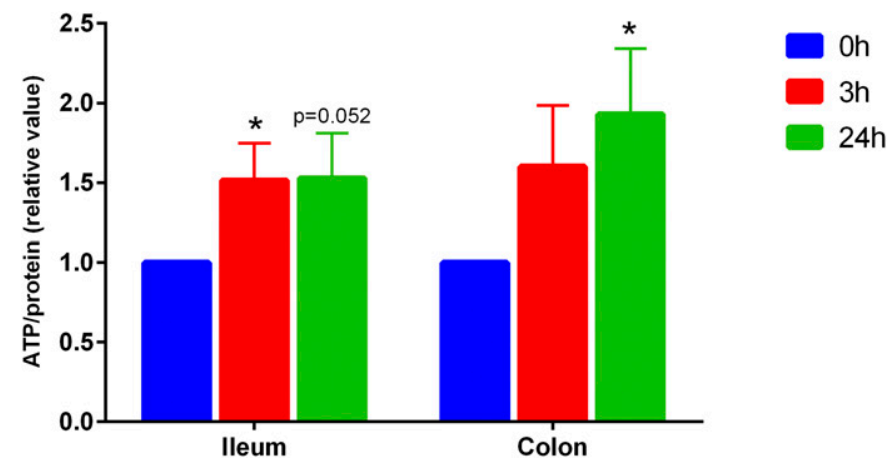

Fig. 2. Viability (relative value) of human ileum and colon slices $(n=7)$. Data are expressed as mean \pm S.E.M. $* P<0.05$ vs. control $(0$ hour $)$. 


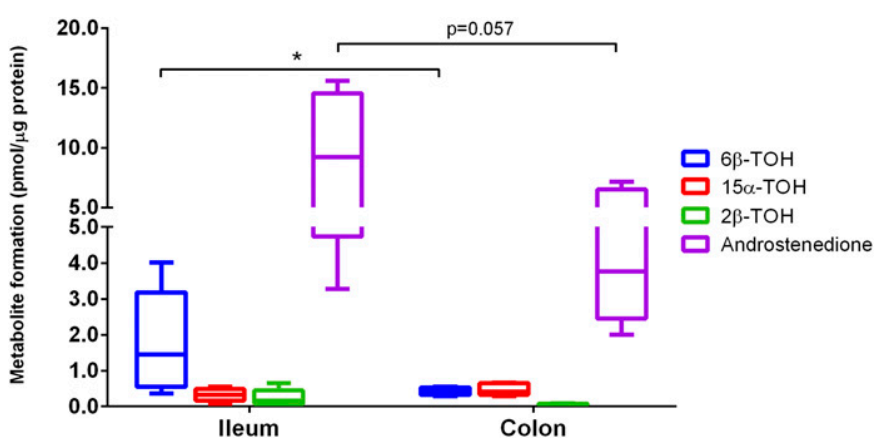

Fig. 3. Testosterone metabolite formation in human ileum and colon slices $(n=5)$. Data are expressed as mean \pm range. $* P<0.05$.

Assessment of Phase II Metabolism in Ileum and Colon. Next, we investigated phase II metabolism by using 7-HC. As shown in Fig. 4, both glucuronidation and sulfation reactions took place in the ileum and the colon $(n=7)$. Although not statistically significant, the concentration of 7-HC glucuronide was 2.2-fold higher in the colon compared with the ileum. In contrast, there was no statistically significant difference in sulfation in the ileum and colon. Furthermore, Fig. 4 shows that, in the colon, 7-HC glucuronide levels were 5.8 times higher compared with 7 -HC sulfate $(P<0.05)$. Taken together, these results suggest that phase II reactions are more prevalent in the colon than the ileum, especially regarding glucuronidation.

Furthermore, we studied the overall occurrence of phase I and II metabolism in the ileum and colon by comparing relative metabolite formation. A ratio higher than one $(r>1)$ indicates that the metabolites were more prevalent in the ileum than the colon. Conversely, a ratio of less than $1(r<1)$ shows that the metabolites were present mainly in the colon instead of the ileum. As shown in Fig. 5, androstenedione, $6 \beta-\mathrm{TOH}, 2 \beta-\mathrm{TOH}$, and 7-HC sulfate, were predominantly found in the ileum instead of the colon. While $15 \alpha-\mathrm{TOH}(0.69)$, and $7-\mathrm{HC}$ glucuronide $(0.45)$ levels were higher in the colon rather than the ileum (Supplemental Table S1).

Moreover, as illustrated in Fig. 6, we also observed sex differences in phase II metabolite formation, which appeared to be higher in men compared with women.

Influence of Viability on Metabolite Formation. To exclude the possibility that metabolite formation was influenced by the viability of the slices we performed a correlation analysis, which revealed no significant correlation between the ATP content of the slices and the formation of most phase I and phase II metabolites, both in the ileum and

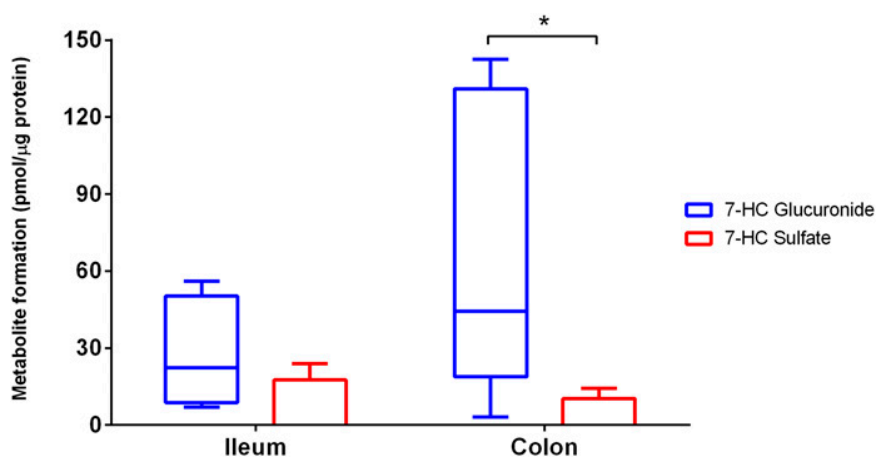

Fig. 4. 7-Hydroxycoumarin metabolite formation in human ileum and colon slices $(n=7)$. Data are expressed as mean \pm S.E.M. $* P<0.05$. Zero metabolite formation due to the limit of detection of the HPLC.

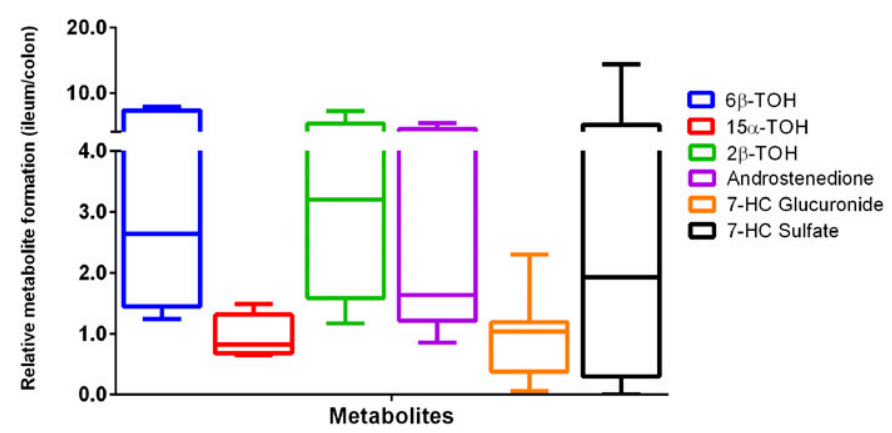

Fig. 5. Testosterone and 7-hydroxycoumarin metabolite formation in human ileum and colon slices $(n=5-7)$. Data are expressed as mean \pm range.

colon. However, we found a trend toward a negative correlation between ATP levels and 7-HC glucuronide in the ileum $(P=0.08, r=0.7)$.

Correlation between Phase I and Phase II Metabolism. Finally, to understand better the relationship between phase I and phase II metabolism, we investigated the correlation between all the studied metabolites.

As demonstrated in Fig. 7, we found a strong positive correlation in the ileum between $2 \beta-\mathrm{TOH}$ and $6 \beta-\mathrm{TOH}, 7-\mathrm{HC}$ glucuronide and $6 \beta$ $\mathrm{TOH}, 7-\mathrm{HC}$ sulfate and $6 \beta-\mathrm{TOH}, 7-\mathrm{HC}$ glucuronide and $2 \beta-\mathrm{TOH}$, as well as 7 -HC sulfate and $2 \beta$-TOH (Supplemental Table S2). There was also a strong positive correlation between 7 -HC glucuronide and $15 \alpha-\mathrm{TOH}$, as well as 7-HC sulfate and 7-HC glucuronide in the colon (Supplemental Table S2). However, we did not observe a correlation

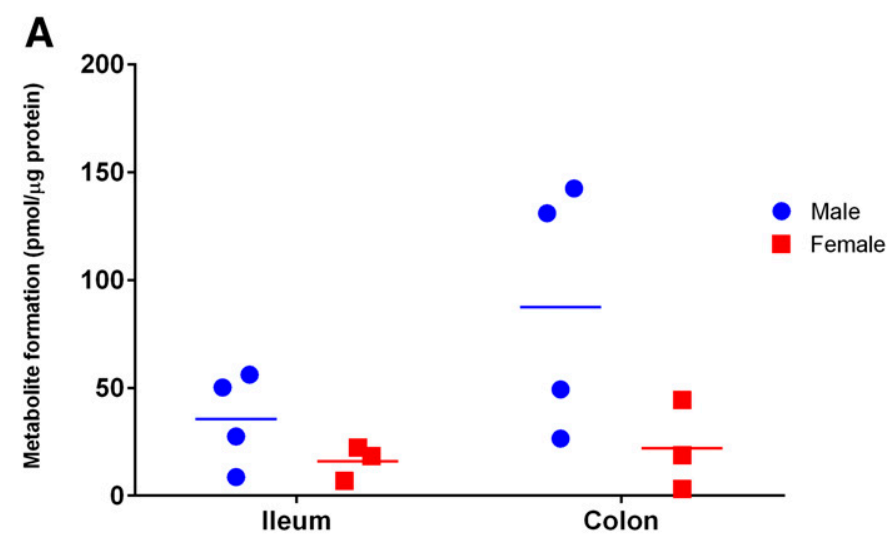

B

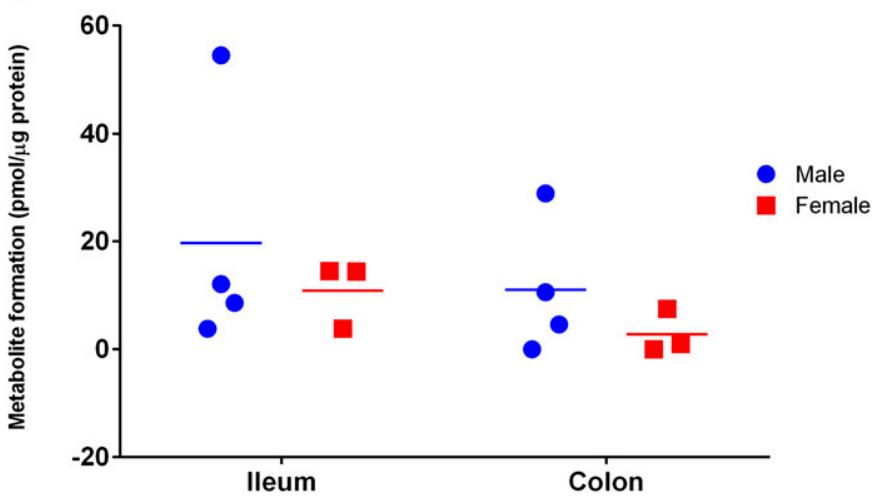

Fig. 6. Sex differences on phase II metabolism of human ileum and colon slices: (A) 7-HC glucuronide; (B) 7-HC sulfate. Data are expressed as mean \pm range. 


\section{Ileum}

A

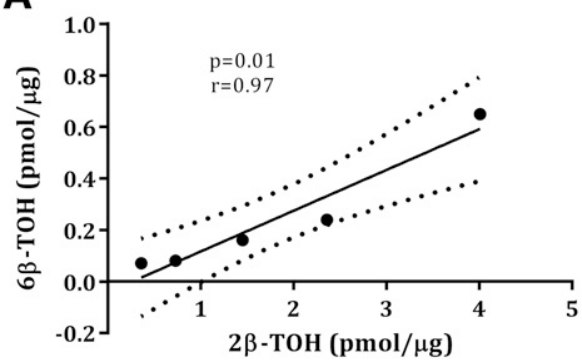

C

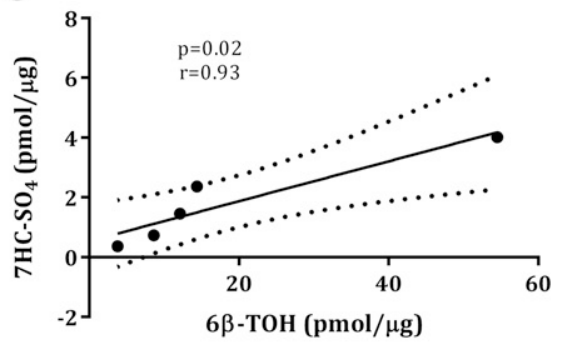

E

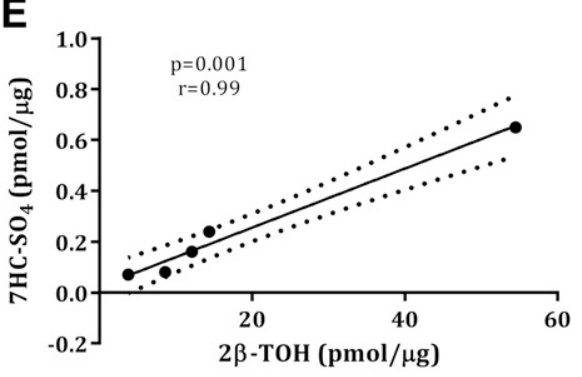

B

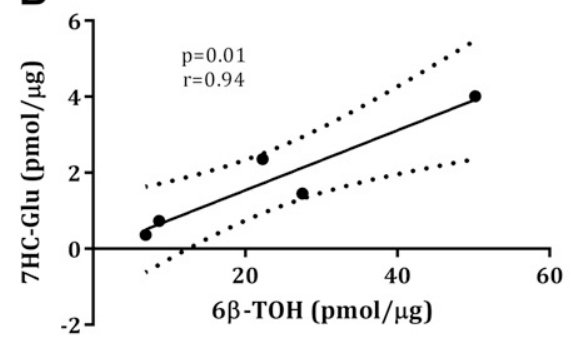

D

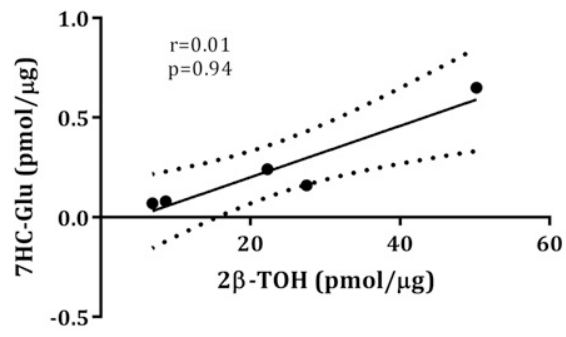

Fig. 7. Correlations of phase I and phase II metabolite formation in the ileum (A-E) and colon (F-G), $n=5-7 . P<0.05, r> \pm 0.5$.

\section{Colon}

$\mathbf{F}$

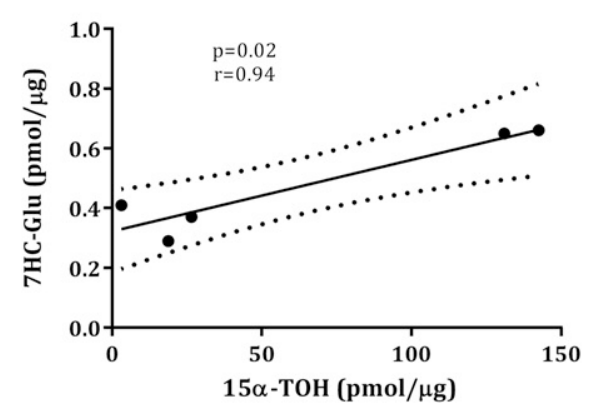

G

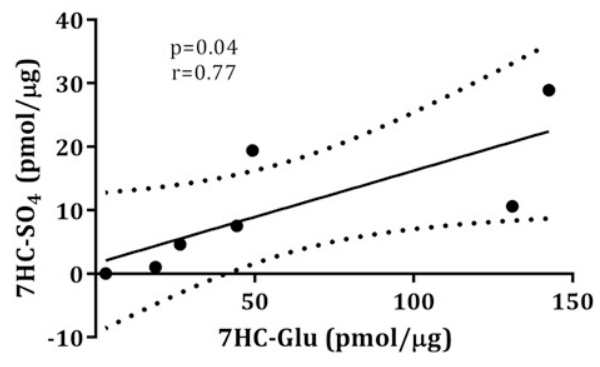

between the metabolic activity of phase I and phase II enzymes in matched slices (Supplemental Figs. S1 and S2).

\section{Discussion}

The intestines fulfill an important role in drug metabolism, and it is known that the expression levels of the involved enzymes differ along the intestinal tract. However, studies in humans are lacking, especially with regards to intraindividual differences in regional metabolism. Here, we evaluated the differences in phase I and II metabolism using matched ileum and colon hPCIS.

Our results demonstrated that the ATP level, as a marker of viability, increased after 3 hours of culture and remained elevated for 24 hours in both human ileum and colon PCIS. A similar observation was previously made using rat PCIS (van de Kerkhof et al., 2007). In addition, we found no correlation between ATP levels and the formation of phase I and phase II metabolites, in both ileum and colon (Supplemental Figs. S3 and S4). This result emphasizes that the viability of PCIS had no impact on the metabolic activity of phase I and phase II enzymes. 
A unique aspect of this study was the fact that we were able to use matched ileum and colon hPCIS. This allowed us to show that there is no correlation between the metabolic rate of phase I and phase II enzymes in matched slices (Supplemental Figs. S1 and S2). This strongly suggests that the regional drug metabolism capacity is differently regulated within one human being. Furthermore, pharmacokinetics and bioavailability of drugs are greatly affected in patients with a short small bowel following gastrointestinal surgery, and the changes will vastly differ between patients (Severijnen et al., 2004; Titus et al., 2013). Studies with matched hPCIS can aid in the identification of suitable therapies for this patient population.

We demonstrated that testosterone metabolism mostly occurred in the ileum. This result is in line with a previous study showing that protein levels of CYP3A, which exhibits testosterone $6 \beta$-hydroxylase activity (Emoto et al., 2000b), is highest in the proximal region of the intestine and declines in the distal part (McKinnon et al., 1995; Paine et al., 1997; Xie et al., 2016; Drozdzik et al., 2018). In addition, other studies revealed that $\mathrm{CYP} 3 \mathrm{~A}$ and $\mathrm{CYP} 2 \mathrm{C}$ are the major intestinal CYPs, accounting for approximately $80 \%$ and $18 \%$, respectively, of all CYPs (Thelen and Dressman, 2009; Nakamura et al., 2016). In line with our findings, Thelen and Dressman (2009) also showed that CYP3A content is generally higher in the proximal region of the intestine.

Our results further demonstrated that metabolism of 7-HC mostly took place in the colon. These findings corroborate previous works showing that UGTs are primarily found in the colon (Pacifici et al., 1989; Zhang et al., 2018). In contrast, Drozdzik et al. (2018) reported that UGT1A1 was the most abundant UGT in jejunum compared with other intestinal segments. However, they only measured mRNA level and protein content, not enzyme activity.

Previously, the metabolic activity in intestinal slices and intestinal rat microsomes has been compared. The results showed that the metabolic rates in intestinal slices were significantly higher for all substrates studied (including TT, 3- to 29-fold) compared with the microsomes (Martignoni et al., 2006). In addition, in agreement with our findings, Ho et al. (2017) described that CYP3A4 (TT as a substrate) was the most active enzyme in cryopreserved enterocytes from human small intestines followed by UGT and SULT (7-HC as a substrate), respectively.

Furthermore, we also discovered a strong correlation between several products formed by CYP3A (e.g., $2 \beta-\mathrm{TOH}$ and $6 \beta$-TOH; Emoto et al., 2000b; Vimercati et al., 2017), CYP2A (15 $\alpha$-TOH; Honkakoski and Negishi, 1997), UGT1A (7-HC glucuronide; Wang et al., 2006), and SULT1 (7-HC sulfate, Wang et al., 2006) in both the ileum and colon. These results suggest that there might be a shared regulatory mechanism between phase I and phase II enzymes. Indeed, work by Xie and colleagues demonstrated that the constitutive androstane receptor and pregnane $\mathrm{X}$ receptor are involved in the regulation of CYP3A and CYP2B (Xie et al., 2000), as well as specific UGT1A isoforms (Xie et al., 2003). In addition, Maglich et al. (2003) showed that constitutive androstane receptor regulates CYP2A6 and SULT1. Thus, it is clear that several signaling pathways can regulate both phase I and phase II enzymes.

We also observed that the rate of phase II metabolism was higher in male PCIS compared with female PCIS. To the best of our knowledge, sex differences in human intestinal metabolism have not been reported before. However, using human liver microsomes, Court et al. (2001) previously established that acetaminophen metabolism, catalyzed by UGT1A1, UGT1A6, and UGT1A9, was higher in men compared with women. Men also show a higher rate of clearance of several benzodiazepines (e.g., diazepam, chlordiazepoxide, and olanzapine), which is catalyzed by CYP1A2. Furthermore, men more rapidly clear chloroxazone, a CYP2E1 substrate (Waxman and Holloway, 2009). Also, it is clear that endogenous sex hormones can impact hepatic enzyme activity, resulting in sex differences in pharmacokinetics (Waxman and Holloway, 2009). However, Miyauchi et al. (2016) reported that there was no significant difference between men and women in the expression level of CYPs and UGTs in human jejunal tissues. In addition, Her et al. (1996) described a large variation in the jejunal expression of SULT1E1 and SULT2A1 independent of sex or age. Thus more research is needed to characterize fully sex-based differences in intestinal drug metabolism.

In conclusion, PCIS is a promising model to investigate intestinal drug metabolism. Based on this study, it can be concluded that there is a significant difference in the rate of phase I and II metabolism in the ileum and the colon. We demonstrated that phase I metabolism predominantly occurs in ileum PCIS, while phase II metabolism mostly takes place in colon PCIS. Moreover, our study revealed that PCIS could be used to investigate sex differences in drug metabolism.

\section{Authorship Contributions}

Participated in research design: Hofker, Mutsaers, Olinga.

Conducted experiments: Iswandana, Irianti.

Contributed new reagents or analytic tools: Oosterhuis, Merema, de Jager.

Performed data analysis: Iswandana, Irianti, Mutsaers, Olinga.

Wrote or contributed to the writing of the manuscript: Iswandana, Mutsaers, Olinga.

\section{References}

Arlotto MP, Trant JM, and Estabrook RW (1991) Measurement of steroid hydroxylation reactions by high-performance liquid chromatography as indicator of P450 identity and function. Methods Enzymol 206:454-462.

Busch D, Fritz A, Partecke LI, Heidecke CD, and Oswald S (2018) LC-MS/MS method for the simultaneous quantification of intestinal CYP and UGT activity. J Pharm Biomed Anal 155: 194-201.

Chhabra RS (1979) Intestinal absorption and metabolism of xenobiotics. Environ Health Perspect 33:61-69.

Choi MH, Skipper PL, Wishnok JS, and Tannenbaum SR (2005) Characterization of testosterone $11 \beta$-hydroxylation catalyzed by human liver microsomal cytochromes P450. Drug Metab Dispos 33:714-718.

Court MH, Duan SX, von Moltke LL, Greenblatt DJ, Patten CJ, Miners JO, and Mackenzie PI (2001) Interindividual variability in acetaminophen glucuronidation by human liver microsomes: identification of relevant acetaminophen UDP-glucuronosyltransferase isoforms. J Pharmacol Exp Ther 299:998-1006.

de Graaf IAM, Olinga P, de Jager MH, Merema MT, de Kanter R, van de Kerkhof EG, and Groothuis GMM (2010) Preparation and incubation of precision-cut liver and intestinal slices for application in drug metabolism and toxicity studies. Nat Protoc 5:1540-1551.

De Kanter R, De Jager MH, Draaisma AL, Jurva JU, Olinga P, Meijer DKF, and Groothuis GMM (2002) Drug-metabolizing activity of human and rat liver, lung, kidney and intestine slices. Xenobiotica 32:349-362.

de Kanter R, Tuin A, van de Kerkhof E, Martignoni M, Draaisma AL, de Jager MH, de Graaf IA, Meijer DKF, and Groothuis GMM (2005) A new technique for preparing precision-cut slices from small intestine and colon for drug biotransformation studies. J Pharmacol Toxicol Methods 51:65-72.

de Waziers I, Cugnenc PH, Yang CS, Leroux JP, and Beaune PH (1990) Cytochrome P 450 isoenzymes, epoxide hydrolase and glutathione transferases in rat and human hepatic and extrahepatic tissues. J Pharmacol Exp Ther 253:387-394.

Ding X and Kaminsky LS (2003) Human extrahepatic cytochromes P450: function in xenobiotic metabolism and tissue-selective chemical toxicity in the respiratory and gastrointestinal tracts. Aпnи Rev Pharmacol Toxicol 43:149-173.

Doherty MM and Charman WN (2002) The mucosa of the small intestine: how clinically relevant as an organ of drug metabolism? Clin Pharmacokinet 41:235-253.

Drozdzik M, Busch D, Lapczuk J, Müller J, Ostrowski M, Kurzawski M, and Oswald S (2018) Protein abundance of clinically relevant drug-metabolizing enzymes in the human liver and intestine: a comparative analysis in paired tissue specimens. Clin Pharmacol Ther 104:515-524. Emoto C, Yamazaki H, Yamasaki S, Shimada N, Nakajima M, and Yokoi T (2000a) Characterization of cytochrome P450 enzymes involved in drug oxidations in mouse intestinal microsomes. Xenobiotica 30:943-953.

Emoto C, Yamazaki H, Yamasaki S, Shimada N, Nakajima M, and Yokoi T (2000b) Use of everted sacs of mouse small intestine as enzyme sources for the study of drug oxidation activities in vitro. Xenobiotica 30:971-982.

Glaeser H, Drescher S, Hofmann U, Heinkele G, Somogyi AA, Eichelbaum M, and Fromm MF (2004) Impact of concentration and rate of intraluminal drug delivery on absorption and gut wall metabolism of verapamil in humans. Clin Pharmacol Ther 76:230-238.

Gröer C, Busch D, Patrzyk M, Beyer K, Busemann A, Heidecke CD, Drozdzik M, Siegmund W, and Oswald S (2014) Absolute protein quantification of clinically relevant cytochrome P450 enzymes and UDP-glucuronosyltransferases by mass spectrometry-based targeted proteomics. $J$ Pharm Biomed Anal 100:393-401.

Her C, Szumlanski C, Aksoy IA, and Weinshilboum RM (1996) Human jejunal estrogen sulfotransferase and dehydroepiandrosterone sulfotransferase: immunochemical characterization of individual variation. Drug Metab Dispos 24:1328-1335.

Ho MD, Ring N, Amaral K, Doshi U, and Li AP (2017) Human enterocytes as an in vitro model for the evaluation of intestinal drug metabolism: characterization of drug-metabolizing enzyme 
activities of cryopreserved human enterocytes from twenty-four donors. Drug Metab Dispos 45 : 686-691.

Honkakoski P and Negishi M (1997) The structure, function, and regulation of cytochrome P450 2A enzymes. Drug Metab Rev 29:977-996.

Kim S, Thiessen PA, Bolton EE, Chen J, Fu G, Gindulyte A, Han L, He J, He S, Shoemaker BA, et al (2016) PubChem substance and compound databases. Nucleic Acids Res 44 (D1):D1202-D1213.

Maglich JM, Parks DJ, Moore LB, Collins JL, Goodwin B, Billin AN, Stoltz CA, Kliewer SA, Lambert MH, Willson TM, et al. (2003) Identification of a novel human constitutive androstane receptor (CAR) agonist and its use in the identification of CAR target genes. J Biol Chem 278 17277-17283.

Martignoni M, Groothuis G, and de Kanter R (2006) Comparison of mouse and rat cytochrome P450-mediated metabolism in liver and intestine. Drug Metab Dispos 34:1047-1054.

McKinnon RA, Burgess WM, Hall PM, Roberts-Thomson SJ, Gonzalez FJ, and McManus ME (1995) Characterisation of CYP3A gene subfamily expression in human gastrointestinal tissues. Gut 36:259-267.

Miyauchi E, Tachikawa M, Declèves X, Uchida Y, Bouillot JL, Poitou C, Oppert JM, Mouly S, Bergmann JF, Terasaki T, et al. (2016) Quantitative atlas of cytochrome P450, UDPglucuronosyltransferase, and transporter proteins in jejunum of morbidly obese subjects. $M o$ Pharm 13:2631-2640.

Nakamura K, Hirayama-Kurogi M, Ito $S$, Kuno T, Yoneyama T, Obuchi W, Terasaki T, and Ohtsuki S (2016) Large-scale multiplex absolute protein quantification of drug-metabolizing enzymes and transporters in human intestine, liver, and kidney microsomes by SWATH-MS: comparison with MRM/SRM and HR-MRM/PRM. Proteomics 16:2106-2117.

Pacifici GM, Franchi M, Gervasi PG, Longo V, di Simplicio P, Temellini A, and Giuliani L (1989) Profile of drug-metabolizing enzymes in human ileum and colon. Pharmacology 38:137-145.

Paine MF, Khalighi M, Fisher JM, Shen DD, Kunze KL, Marsh CL, Perkins JD, and Thummel KE (1997) Characterization of interintestinal and intraintestinal variations in human CYP3Adependent metabolism. J Pharmacol Exp Ther 283:1552-1562.

Peters SA, Jones CR, Ungell AL, and Hatley OJD (2016) Predicting drug extraction in the human gut wall: assessing contributions from drug metabolizing enzymes and transporter proteins using preclinical models. Clin Pharmacokinet 55:673-696.

Pham BT, van Haaften WT, Oosterhuis D, Nieken J, de Graaf IA, and Olinga P (2015) Precisioncut rat, mouse, and human intestinal slices as novel models for the early-onset of intestinal fibrosis. Physiol Rep 3:e12323.

Poosti F, Pham BT, Oosterhuis D, Poelstra K, van Goor H, Olinga P, and Hillebrands JL (2015) Precision-cut kidney slices (PCKS) to study development of renal fibrosis and efficacy of drug targeting ex vivo. Dis Model Mech 8:1227-1236.

Severijnen R, Bayat N, Bakker H, Tolboom J, and Bongaerts G (2004) Enteral drug absorption in patients with short small bowel : a review. Clin Pharmacokinet 43:951-962.

Thelen K and Dressman JB (2009) Cytochrome P450-mediated metabolism in the human gut wall J Pharm Pharmacol 61:541-558.

Titus R, Kastenmeier A, and Otterson MF (2013) Consequences of gastrointestinal surgery on drug absorption. Nutr Clin Pract 28:429-436.

van de Kerkhof EG, de Graaf IAM, de Jager MH, and Groothuis GMM (2007) Induction of phase I and II drug metabolism in rat small intestine and colon in vitro. Drug Metab Dispos 35:898-907. van de Kerkhof EG, de Graaf IA, de Jager MH, Meijer DK, and Groothuis GM (2005) Characterization of rat small intestinal and colon precision-cut slices as an in vitro system for drug metabolism and induction studies. Drug Metab Dispos 33:1613-1620.

van de Kerkhof EG, de Graaf IA, Ungell AL, and Groothuis GM (2008) Induction of metabolism and transport in human intestine: validation of precision-cut slices as a tool to study induction of drug metabolism in human intestine in vitro. Drug Metab Dispos 36:604-613.

van de Kerkhof EG, Ungell AL, Sjöberg AK, de Jager MH, Hilgendorf C, de Graaf IA, and Groothuis GM (2006) Innovative methods to study human intestinal drug metabolism in vitro: precision-cut slices compared with ussing chamber preparations. Drug Metab Dispos 34: 1893-1902.

Vimercati S, Büchi M, Zielinski J, Peduto N, and Mevissen M (2017) Testosterone metabolism of equine single CYPs of the 3A subfamily compared to the human CYP3A4. Toxicol In Vitro 41: $83-91$.

von Richter O, Greiner B, Fromm MF, Fraser R, Omari T, Barclay ML, Dent J, Somogyi AA, and Eichelbaum M (2001) Determination of in vivo absorption, metabolism, and transport of drugs by the human intestinal wall and liver with a novel perfusion technique. Clin Pharmacol Ther 70:217-227.

Wang DQ, Tazuma S, Cohen DE, and Carey MC (2003) Feeding natural hydrophilic bile acids inhibits intestinal cholesterol absorption: studies in the gallstone-susceptible mouse. Am J Physiol Gastrointest Liver Physiol 285:G494-G502.

Wang Q, Ye C, Jia R, Owen AJ, Hidalgo IJ, and Li J (2006) Inter-species comparison of 7-hydroxycoumarin glucuronidation and sulfation in liver S9 fractions. In Vitro Cell Dev Biol Anim 42:8-12.

Waxman DJ and Holloway MG (2009) Sex differences in the expression of hepatic drug metabolizing enzymes. Mol Pharmacol 76:215-228.

Xie F, Ding X, and Zhang QY (2016) An update on the role of intestinal cytochrome P450 enzymes in drug disposition. Acta Pharm Sin B 6:374-383.

Xie W, Barwick JL, Simon CM, Pierce AM, Safe S, Blumberg B, Guzelian PS, and Evans RM (2000) Reciprocal activation of xenobiotic response genes by nuclear receptors SXR/PXR and CAR. Genes Dev 14:3014-3023.

Xie W, Yeuh MF, Radominska-Pandya A, Saini SP, Negishi Y, Bottroff BS, Cabrera GY, Tukey RH, and Evans RM (2003) Control of steroid, heme, and carcinogen metabolism by nuclear pregnane X receptor and constitutive androstane receptor. Proc Natl Acad Sci USA 100: $4150-4155$.

Zhang H, Basit A, Busch D, Yabut K, Bhatt DK, Drozdzik M, Ostrowski M, Li A, Collins C, Oswald S, et al. (2018) Quantitative characterization of UDP-glucuronosyltransferase 2B17 in human liver and intestine and its role in testosterone first-pass metabolism. Biochem Pharmacol 156:32-42.

Address correspondence to: Peter Olinga, Division of Pharmaceutical Technology and Biopharmacy, Department of Pharmacy, University of Groningen, Antonius Deusinglaan 1, 9713 AV, Groningen, The Netherlands. E-mail: p.olinga@rug.nl 